\title{
A Fixed Point Theorem in Orbitally Complete Partially Ordered Metric Spaces
}

\author{
G. V. R. Babu, ${ }^{1}$ P. D. Sailaja, ${ }^{1,2}$ and K. T. Kidane ${ }^{1,3}$ \\ ${ }^{1}$ Department of Mathematics, Andhra University, Visakhapatnam 530 003, India \\ ${ }^{2}$ Department of Mathematics, Lendi Institute of Engineering and Technology, Vizianagaram 535 005, India \\ ${ }^{3}$ Department of Mathematics, Jimma University, Jimma-378, Ethiopia
}

Correspondence should be addressed to G. V. R. Babu; gvr_babu@hotmail.com

Received 14 June 2013; Revised 30 October 2013; Accepted 2 November 2013

Academic Editor: Ram U. Verma

Copyright (c) 2013 G. V. R. Babu et al. This is an open access article distributed under the Creative Commons Attribution License, which permits unrestricted use, distribution, and reproduction in any medium, provided the original work is properly cited.

Let $(X, \preceq)$ be a partially ordered set and $T: X \rightarrow X$ be a mapping. We prove a fixed point theorem for the map $T$ satisfying a contractive condition in orbits, when $X$ is $T$-orbitally complete. Our result extends and generalizes the results of Samet et al. (2013) to partially ordered sets. Also, we generalize the results of Ran and Reurings (2004).

\section{Introduction}

In 1922, Banach [1] established a very fundamental result in fixed point theory, namely, the Banach contraction principle. Large number of generalizations have been made on this principle (we refer to Berinde and Vetro [2], Chatterjea [3], Ćirić [4], and Kannan [5, 6]). The Banach contraction principle forces the map $T$ to be continuous. In 1968, Kannan [5] established a fixed point theorem for a map which need not be continuous. Later in 1971, Reich [7] proved a fixed point theorem through which the Banach contraction principle and Kannan's fixed point theorem are generalized.

Recently, Samet et al. [8] established new fixed point theorems in complete metric spaces that generalize the Banach contraction principle and Reich's and Kannan's fixed point theorems.

Theorem 1 (see [8]). Let $(X, d)$ be a complete metric space, $\varphi$ : $X \rightarrow[0, \infty)$ a lower semicontinuous function, and $T: X \rightarrow$ $X$ a given mapping. Suppose that, for any $0<a<b<\infty$, there exists $0<\gamma(a, b)<1$ such that $a \leq d(x, y)+\varphi(x)+\varphi(y) \leq b$ implies

$$
\begin{aligned}
& d(T x, T y)+\varphi(T x)+\varphi(T y) \\
& \quad \leq \gamma(a, b)[d(x, y)+\varphi(x)+\varphi(y)],
\end{aligned}
$$

for all $x, y \in X$. Then, $T$ has a unique fixed point $x^{*} \in X$. Moreover, one has $\varphi\left(x^{*}\right)=0$.

Theorem 2 (see [8]). Let $(X, d)$ be a complete metric space, $\varphi: X \rightarrow[0, \infty)$ a lower semi-continuous function and $T:$ $X \rightarrow X$ a given mapping. Suppose that there exists a constant $\gamma \in(0,1 / 2)$ such that

$$
\begin{aligned}
d(T x, T y)+\varphi(T x)+\varphi(T y) \\
\leq \gamma[d(x, T x)+d(y, T y)+\varphi(x)+\varphi(y) \\
\quad+\varphi(T x)+\varphi(T y)],
\end{aligned}
$$

for all $x, y \in X$. Then, $T$ has a unique fixed point $x^{*} \in X$. Moreover, one has $\varphi\left(x^{*}\right)=0$.

Theorem 3 (see [8]). Let $(X, d)$ be a complete metric space, $\varphi$ : $X \rightarrow[0, \infty)$ a lower semi-continuous function and $T: X \rightarrow$ $X$ a given mapping. Suppose that, there exist $\alpha, \beta, \gamma \in(0,1)$ with $\alpha+\beta+\gamma<1$ such that for all $x, y \in X$,

$$
\begin{aligned}
& d(T x, T y)+\varphi(T x)+\varphi(T y) \\
& \quad \leq \alpha[d(x, y)+\varphi(x)+\varphi(y)]
\end{aligned}
$$




$$
\begin{aligned}
& +\beta[d(x, T x)+\varphi(x)+\varphi(T x)] \\
& +\gamma[d(y, T y)+\varphi(y)+\varphi(T y)] .
\end{aligned}
$$

Then, $T$ has a unique fixed point $x^{*} \in X$. Moreover, one has $\varphi\left(x^{*}\right)=0$.

Establishing the existence of fixed points in partially ordered sets is of recent interest in fixed point theory. In 2004, Ran and Reurings [9] established the existence of fixed points for contraction maps in partially ordered sets.

Definition 4 . Let $(X, \preceq)$ be a partially ordered set. A map $T$ : $X \rightarrow X$ is said to be non-decreasing if, for any $x, y \in X$ with $x \preceq y$, one has $T x \leq T y$.

Theorem 5 (see [9]). Let $(X, \preceq)$ be a partially ordered set and $d$ be a metric on $X$ such that $(X, d)$ is a complete metric space. Suppose that $T: X \rightarrow X$ is a continuous map and there exists $k \in(0,1)$ such that

$$
d(T x, T y) \leq k d(x, y),
$$

for each $x, y \in X$ with $x \preceq y$. If there exists $x_{0} \in X$ such that $x_{0} \leq T x_{0}$, then $T$ has a fixed point in $X$.

Definition 6. Let $X$ be a nonempty set and $T: X \rightarrow$ $X$. Let $x_{0} \in X$. The orbit of $x_{0}$ is defined by $\mathcal{O}\left(x_{0}\right)=$ $\left\{x_{0}, T x_{0}, T^{2} x_{0}, \ldots\right\}$.

Definition 7. Let $(X, d)$ be a metric space and $T: X \rightarrow X . X$ is said to be T-orbitally complete if every Cauchy sequence in $\mathcal{O}(x), x \in X$, converges to a point in $X$.

Definition 8. Let $(X, d)$ be a metric space and $T: X \rightarrow X$. $T$ is said to be orbitally continuous at $z \in X$ if $T x_{n} \rightarrow T z$ as $n \rightarrow \infty$ whenever $x_{n} \rightarrow z$ as $n \rightarrow \infty$.

If $T$ is continuous at $z \in X$, then $T$ is orbitally continuous at $z$. But its converse need not be true.

For more literature on these concepts, we refer to Türkoğlu et al. [10] and for works on ordered orbitally complete metric spaces we refer to Ding et al. [11] and Nashine et al. [12].

Definition 9. Let $X$ be a nonempty set. Let $\left\{T_{i} / T_{i}: X \rightarrow\right.$ $X, i=1,2,3, \ldots\}$ be a sequence of self-maps. An element $x \in$ $X$ is said to be a common fixed point of $\left\{T_{i}\right\}$ if $T_{i}(x)=x$ for each $i=1,2,3, \ldots$.

In this paper, we prove a fixed point theorem in partially ordered metric space $X$, for a self-map $T: X \rightarrow X$, when $X$ is $T$-orbitally complete. Our result extends and generalizes the results of Samet et al. [8] to partially ordered sets. Our result also generalizes the results of Ran and Reurings [9].

We use the following lemma in our main result.

Lemma 10 (see [13]). Suppose that $(X, d)$ is a metric space. Let $\left\{x_{n}\right\}$ be a sequence in $X$ such that $d\left(x_{n}, x_{n+1}\right) \rightarrow 0$ as $n \rightarrow \infty$. If $\left\{x_{n}\right\}$ is not a Cauchy sequence, then there exist $\epsilon>0$ and sequences of the positive integers $m(k)$ and $n(k)$ with $m(k)>$ $n(k)>k$ such that $d\left(x_{m(k)}, x_{n(k)}\right) \geq \epsilon, d\left(x_{m(k)-1}, x_{n(k)}\right)<\epsilon$ and $\lim _{k \rightarrow \infty} d\left(x_{m(k)}, x_{n(k)}\right)=\epsilon$ and $\lim _{k \rightarrow \infty} d\left(x_{m(k)+1}, x_{n(k)+1}\right)=$ $\epsilon$.

\section{Main Result}

Throughout this paper, we denote that

$$
\begin{gathered}
\Psi=\{\psi:[0, \infty) \longrightarrow[0, \infty): \psi \\
\text { is non-decreasing, continuous and } \\
\psi(t)=0 \Leftrightarrow t=0\} .
\end{gathered}
$$

Theorem 11. Let $(X, \preceq)$ be a partially ordered set and $d a$ metric on $X$. Suppose that $T: X \rightarrow X$ is a non-decreasing map and $x_{0} \in X$ such that $x_{0} \leq T x_{0}$. Suppose that there exist a lower semicontinuous function $\varphi: X \rightarrow[0, \infty)$ and $\psi \in \Psi$ such that the following condition holds.

"For each $0 \leq a<b<\infty$, there exists $\gamma(a, b) \in[0,1)$ such that $a \leq \psi(d(x, y))+\varphi(x)+\varphi(y) \leq b$ implies

$$
\psi(d(T x, T y))+\varphi(T x)+\varphi(T y) \leq \gamma(a, b) M(x, y),
$$

where

$$
\begin{array}{r}
M(x, y)=\max \{\psi(d(x, y))+\varphi(x)+\varphi(y), \\
\psi(d(x, T x))+\varphi(x)+\varphi(T x), \\
\psi(d(y, T y))+\varphi(y)+\varphi(T y)\}
\end{array}
$$

for each $x, y \in \overline{\mathcal{O}\left(x_{0}\right)}$ with $x \preceq y$."

Assume that $X$ is T-orbitally complete. Then, the sequence $\left\{x_{n}\right\}$ defined by $x_{n+1}=T x_{n}, n=0,1,2, \ldots$, is Cauchy in X. Let $\lim _{n \rightarrow \infty} x_{n}=z, z \in X$. Suppose that either

(a) $T$ is orbitally continuous at $z$

$$
\text { or }
$$

(b) if $\left\{x_{n}\right\}$ is a non-decreasing sequence converging to $x \in$ $X$, then $x_{n} \leq x$, for all $n$.

Then, $z$ is a fixed point of $T$ and $\varphi(z)=0$.

Proof. Let $x_{0} \in X$ such that $x_{0} \preceq T x_{0}$. Choose $x_{1} \in X$ such that $x_{1}=T x_{0}$. Then, $x_{0} \preceq x_{1}$. Since $T$ is non-decreasing, we have $x_{1}=T x_{0} \leq T x_{1}$. Now, we choose $x_{2} \in X$ such that $x_{2}=T x_{1}$. Hence, $x_{1}=T x_{0} \preceq T x_{1}=x_{2}$.

Continuing the same procedure, we obtain a sequence $\left\{x_{n}\right\} \in \overline{\mathcal{O}\left(x_{0}\right)}$ such that

$$
\begin{gathered}
x_{n+1}=T x_{n}, \quad n=0,1,2, \ldots, \\
x_{0} \preceq x_{1} \preceq x_{2} \preceq \cdots \preceq x_{n} \preceq x_{n+1} \preceq \cdots .
\end{gathered}
$$

If there exists an integer $n_{0}$ such that $x_{n_{0}}=x_{n_{0}+1}$, then, $x_{n_{0}}$ is a fixed point of $T$ and hence we are through. Without loss of generality, we assume that $x_{n} \neq x_{n+1}$ for each $n$. 
We denote

$$
\begin{aligned}
u_{n}= & \psi\left(d\left(x_{n}, x_{n+1}\right)\right)+\varphi\left(x_{n}\right) \\
& +\varphi\left(x_{n+1}\right) \quad \text { for each } n=0,1,2, \ldots
\end{aligned}
$$

We show that $u_{n+1} \leq u_{n}$ for each $n=0,1,2, \ldots$. Suppose, if possible, there exists $n_{0}$ such that $u_{n_{0}}<u_{n_{0}+1}$. That is,

$$
\begin{aligned}
0<u_{n_{0}} & =\psi\left(d\left(x_{n_{0}}, x_{n_{0}+1}\right)\right)+\varphi\left(x_{n_{0}}\right)+\varphi\left(x_{n_{0}+1}\right) \\
& <\psi\left(d\left(x_{n_{0}+1}, x_{n_{0}+2}\right)\right)+\varphi\left(x_{n_{0}+1}\right)+\varphi\left(x_{n_{0}+2}\right) \\
& =u_{n_{0}+1} .
\end{aligned}
$$

Then, from (6), there exists $\gamma\left(u_{n_{0}}, u_{n_{0}+1}\right) \in[0,1)$ such that

$$
\begin{aligned}
& \psi\left(d\left(T x_{n_{0}}, T x_{n_{0}+1}\right)\right)+\varphi\left(T x_{n_{0}}\right)+\varphi\left(T x_{n_{0}+1}\right) \\
& \quad \leq \gamma\left(u_{n_{0}}, u_{n_{0}+1}\right) M\left(x_{n_{0}}, x_{n_{0}+1}\right),
\end{aligned}
$$

where

$$
\begin{aligned}
& M\left(x_{n_{0}}, x_{n_{0}+1}\right) \\
& =\max \left\{\psi\left(d\left(x_{n_{0}}, x_{n_{0}+1}\right)\right)+\varphi\left(x_{n_{0}}\right)+\varphi\left(x_{n_{0}+1}\right),\right. \\
& \psi\left(d\left(x_{n_{0}}, x_{n_{0}+1}\right)\right)+\varphi\left(x_{n_{0}}\right)+\varphi\left(x_{n_{0}+1}\right), \\
& \left.\psi\left(d\left(x_{n_{0}+1}, x_{n_{0}+2}\right)\right)+\varphi\left(x_{n_{0}+1}\right)+\varphi\left(x_{n_{0}+2}\right)\right\} \\
& =\max \left\{u_{n_{0}}, u_{n_{0}+1}\right\} \\
& =u_{n_{0}+1} .
\end{aligned}
$$

Hence, from (11), we get

$$
\begin{aligned}
& \psi\left(d\left(T x_{n_{0}}, T x_{n_{0}+1}\right)\right)+\varphi\left(T x_{n_{0}}\right)+\varphi\left(T x_{n_{0}+1}\right) \\
& \quad \leq \gamma\left(u_{n_{0}}, u_{n_{0}+1}\right) u_{n_{0}+1}
\end{aligned}
$$

that is,

$$
u_{n_{0}+1} \leq \gamma\left(u_{n_{0}}, u_{n_{0}+1}\right) u_{n_{0}+1},
$$

a contradiction since $\gamma\left(u_{n_{0}}, u_{n_{0}+1}\right) \in[0,1)$. Therefore, $u_{n+1} \leq$ $u_{n}$ for each $n=0,1,2, \ldots$. That is, the sequence $\left\{u_{n}\right\}$ is a decreasing sequence of real numbers. Hence, there exists $u \geq$ 0 such that $\lim _{n \rightarrow \infty} u_{n}=u$ and $u \leq u_{n}$ for each $n=0,1,2, \ldots$

We now show that $u=0$. Suppose that $u>0$. Since $0<$ $u \leq u_{n} \leq u_{0}$ for all $n$, from (6), there exists $\gamma\left(0, u_{0}\right) \in[0,1)$ such that

$$
\begin{aligned}
u_{n+1} & =\psi\left(d\left(T x_{n}, T x_{n+1}\right)\right)+\varphi\left(T x_{n}\right)+\varphi\left(T x_{n+1}\right) \\
& \leq \gamma\left(0, u_{0}\right) M\left(x_{n}, x_{n+1}\right),
\end{aligned}
$$

where

$$
\begin{aligned}
& M\left(x_{n}, x_{n+1}\right) \\
& =\max \left\{\psi\left(d\left(x_{n}, x_{n+1}\right)\right)+\varphi\left(x_{n}\right)+\varphi\left(x_{n+1}\right),\right. \\
& \psi\left(d\left(x_{n}, x_{n+1}\right)\right)+\varphi\left(x_{n}\right)+\varphi\left(x_{n+1}\right), \\
& \left.\quad \psi\left(d\left(x_{n+1}, x_{n+2}\right)\right)+\varphi\left(x_{n+1}\right)+\varphi\left(x_{n+2}\right)\right\} \\
& =\max \left\{u_{n}, u_{n+1}\right\} \\
& =u_{n} .
\end{aligned}
$$

Thus, from (15), we get

$$
u_{n+1} \leq \gamma\left(0, u_{0}\right) u_{n}
$$

Letting $n \rightarrow \infty$, we get

$$
u \leq \gamma\left(0, u_{0}\right) u
$$

a contradiction. Hence, $u=0$.

Therefore

$$
\lim _{n \rightarrow \infty} u_{n}=\lim _{n \rightarrow \infty}\left[\psi\left(d\left(x_{n}, x_{n+1}\right)\right)+\varphi\left(x_{n}\right)+\varphi\left(x_{n+1}\right)\right]=0 .
$$

Thus, we have $\lim _{n \rightarrow \infty} \psi\left(d\left(x_{n}, x_{n+1}\right)\right)=0$ and $\lim _{n \rightarrow \infty} \varphi\left(x_{n}\right)=0$.

By the property of $\psi$, we have

$$
\lim _{n \rightarrow \infty} d\left(x_{n}, x_{n+1}\right)=0 .
$$

We now show that $\left\{x_{n}\right\} \subseteq \mathcal{O}\left(x_{0}\right)$ is a Cauchy sequence in $X$. Suppose if possible that $\left\{x_{n}\right\}$ is not a Cauchy sequence in $X$. Then, there exist $\epsilon>0$ and sequences of the positive integers $\{m(k)\}$ and $\{n(k)\}$ with $m(k)>n(k)>k$ such that

$$
d\left(x_{m(k)}, x_{n(k)}\right) \geq \epsilon .
$$

We choose $m(k)$, the least positive integer satisfying (21). Then, we have $m(k)>n(k)>k$ with

$$
d\left(x_{m(k)}, x_{n(k)}\right) \geq \epsilon, \quad d\left(x_{m(k)-1}, x_{n(k)}\right)<\epsilon .
$$

Then, by Lemma 10, we have

$$
\lim _{k \rightarrow \infty} d\left(x_{m(k)}, x_{n(k)}\right)=\epsilon ; \quad \lim _{k \rightarrow \infty} d\left(x_{m(k)+1}, x_{n(k)+1}\right)=\epsilon .
$$

Now, since $\lim _{k \rightarrow \infty} d\left(x_{m(k)}, x_{m(k)-1}\right)=0$, there exists a number $K$ such that

$$
d\left(x_{m(k)}, x_{m(k)-1}\right)<\epsilon \text { for each } k \geq K .
$$

Hence,

$$
\begin{aligned}
d\left(x_{m(k)}, x_{n(k)}\right) & \leq d\left(x_{m(k)}, x_{m(k)-1}\right)+d\left(x_{m(k)-1}, x_{n(k)}\right) \\
& <\epsilon+\epsilon \text { for all } k \geq K \\
& =2 \epsilon \text { for all } k \geq K .
\end{aligned}
$$


Since $\psi$ is non-decreasing, we have

$$
\psi\left(d\left(x_{m(k)}, x_{n(k)}\right)\right) \leq \psi(2 \epsilon) \text { for all } k \geq K .
$$

Now, using (22), we get

$$
\begin{aligned}
0<a=\psi(\epsilon) & \leq \psi\left(d\left(x_{m(k)}, x_{n(k)}\right)\right) \\
& \leq \psi\left(d\left(x_{m(k)}, x_{n(k)}\right)\right)+\varphi\left(x_{m(k)}\right)+\varphi\left(x_{n(k)}\right) \\
& <\psi(2 \epsilon)+u_{m(k)}+u_{n(k)} \quad \text { for each } k \geq K \\
& \leq \psi(2 \epsilon)+u_{0}+u_{0} \quad \text { for each } k \geq K \\
& =\psi(2 \epsilon)+2 u_{0}=b \text { (say) for each } k \geq K
\end{aligned}
$$

That is, $0<a \leq \psi\left(d\left(x_{m(k)}, x_{n(k)}\right)\right)+\varphi\left(x_{m(k)}\right)+\varphi\left(x_{n(k)}\right)<b$ for each $k \geq K$.

Therefore, from (6), there exists $\gamma(a, b) \in[0,1)$ such that

$$
\begin{aligned}
& \psi\left(d\left(x_{m(k)+1}, x_{n(k)+1}\right)\right)+\varphi\left(x_{m(k)+1}\right)+\varphi\left(x_{n(k)+1}\right) \\
& \quad \leq \gamma(a, b) M\left(x_{m(k)}, x_{n(k)}\right),
\end{aligned}
$$

where

$$
\begin{aligned}
& M\left(x_{m(k)}, x_{n(k)}\right) \\
&=\max \left\{\psi\left(d\left(x_{m(k)}, x_{n(k)}\right)\right)+\varphi\left(x_{m(k)}\right)+\varphi\left(x_{n(k)}\right),\right. \\
& \psi\left(d\left(x_{m(k)}, x_{m(k)+1}\right)\right)+\varphi\left(x_{m(k)}\right)+\varphi\left(x_{m(k)+1}\right), \\
&\left.\psi\left(d\left(x_{n(k)}, x_{n(k)+1}\right)\right)+\varphi\left(x_{n(k)}\right)+\varphi\left(x_{n(k)+1}\right)\right\} .
\end{aligned}
$$

Letting $k \rightarrow \infty$, we get

$$
\lim _{k \rightarrow \infty} M\left(x_{m(k)}, x_{n(k)}\right)=\psi(\epsilon) .
$$

Now, letting $k \rightarrow \infty$ in (28), we get

$$
\psi(\epsilon) \leq \gamma(a, b) \psi(\epsilon),
$$

a contradiction. Hence, $\left\{x_{n}\right\} \subseteq \mathcal{O}\left(x_{0}\right)$ is a Cauchy sequence in $X$. Since $X$ is $T$-orbitally complete, there exists $z \in X$ such that $\lim _{n \rightarrow \infty} x_{n}=z$.

Since $\varphi$ is lower semi-continuous, we have

$$
\varphi(z) \leq \liminf _{n \rightarrow \infty} \varphi\left(x_{n}\right)=0
$$

Hence, $\varphi(z)=0$.

Now, suppose that (a) holds. That is, $T$ is orbitally continuous at $z$. Then, $x_{n+1}=T x_{n} \rightarrow T z$. Hence, $T z=z$.

Now, suppose that $(b)$ holds. Then, we have $x_{n} \preceq z$ for all $n$.

If $x_{n}=z$ for infinitely many $n$, then $x_{n+1}=T x_{n}=T z$. But $x_{n+1} \rightarrow z$. Hence, $T z=z$. So, without loss of generality, we assume that $d\left(x_{n}, z\right)>0$ for infinitely many $n$. That is, there exists a subsequence $\left\{x_{n(k)}\right\}$ of $\left\{x_{n}\right\}$ such that $d\left(x_{n(k)}, z\right)>0$ for each $k=0,1,2, \ldots$.
As the sequence $\left\{d\left(x_{n(k)}, z\right)\right\}$ is convergent, it is bounded. Hence, there exists $l \geq 0$ such that $d\left(x_{n(k)}, z\right) \leq l$ for each $k=0,1,2, \ldots$.

Since $\psi$ is non-decreasing, we have

$$
\psi\left(d\left(x_{n(k)}, z\right)\right) \leq \psi(l) \quad \text { for each } k=0,1,2, \ldots
$$

Now, for each $k=0,1,2, \ldots$, we have

$$
\begin{aligned}
0 & \leq \psi\left(d\left(x_{n(k)}, z\right)\right) \\
& \leq \psi\left(d\left(x_{n(k)}, z\right)\right)+\varphi\left(x_{n(k)}\right)+\varphi(z) \\
& <\psi\left(d\left(x_{n(k)}, z\right)\right)+u_{n(k)} \\
& \leq \psi(l)+u_{0}=b \text { (say). }
\end{aligned}
$$

Hence, from (6), there exists $\gamma(0, b) \in[0,1)$ such that

$$
\begin{aligned}
& \psi\left(d\left(x_{n(k)+1}, T z\right)\right)+\varphi\left(x_{n(k)+1}\right)+\varphi(T z) \\
& \quad \leq \gamma(0, b) M\left(x_{n(k)}, z\right),
\end{aligned}
$$

where

$$
\begin{aligned}
& M\left(x_{n(k)}, z\right) \\
& =\max \left\{\psi\left(d\left(x_{n(k)}, z\right)\right)+\varphi\left(x_{n(k)}\right)+\varphi(z),\right. \\
& \psi\left(d\left(x_{n(k)}, x_{n(k)+1}\right)\right)+\varphi\left(x_{n(k)}\right)+\varphi\left(x_{n(k)+1}\right), \\
& \psi(d(z, T z))+\varphi(z)+\varphi(T z)\}
\end{aligned}
$$

and $\lim _{k \rightarrow \infty} M\left(x_{n(k)}, z\right)=\psi(d(z, T z))+\varphi(T z)$, since $\varphi(z)=$ 0 .

Letting $k \rightarrow \infty$ in (35), we get

$$
\psi(d(z, T z))+\varphi(T z) \leq \gamma(a, b)[\psi(d(z, T z))+\varphi(T z)] .
$$

Thus, $\psi(d(z, T z))+\varphi(T z)=0$. That is, $T z=z$.

\section{Corollaries and Examples}

The following are the possible corollaries of Theorem 11.

Corollary 12. Let $(X, \preceq)$ be a partially ordered set. Suppose that $T: X \rightarrow X$ is a non-decreasing selfmap on $X$. Assume that there is a metric $d$ on $X$ such that $X$ is T-orbitally complete. Assume that there exists $x_{0} \in X$ such that $x_{0} \preceq T x_{0}$. Suppose that there exist a lower semi-continuous function $\varphi: X \rightarrow$ $[0, \infty), \psi \in \Psi$, and $k \in[0,1)$ such that

$$
\psi(d(T x, T y))+\varphi(T x)+\varphi(T y) \leq k M(x, y)
$$

where

$$
\begin{array}{r}
M(x, y)=\max \{\psi(d(x, y))+\varphi(x)+\varphi(y), \\
\psi(d(x, T x))+\varphi(x)+\varphi(T x), \\
\psi(d(y, T y))+\varphi(y)+\varphi(T y)\}
\end{array}
$$


for each $x, y \in \overline{\mathcal{O}\left(x_{0}\right)}$ with $x \leq y$.

Then, the sequence $\left\{x_{n}\right\}$ defined by $x_{n+1}=T x_{n}, n=$ $0,1,2, \ldots$, is Cauchy in X. Let $\lim _{n \rightarrow \infty} x_{n}=z$. Suppose that either $(a)$ or $(b)$ of Theorem 11 holds, then $z$ is a fixed point of $T$ and $\varphi(z)=0$.

Corollary 13. Let $(X, \preceq)$ be a partially ordered set. Suppose that $T: X \rightarrow X$ is a non-decreasing selfmap on $X$. Assume that there is a metric $d$ on $X$ such that $X$ is T-orbitally complete metric space. Assume that there exists $x_{0} \in X$ such that $x_{0} \preceq$ $T x_{0}$. Suppose that there exist a lower semi-continuous function $\varphi: X \rightarrow[0, \infty), \psi \in \Psi$ such that the following condition holds.

"for each $0 \leq a<b<\infty$, there exists $\gamma(a, b) \in[0,1)$ such that $a \leq \psi(d(x, y))+\varphi(x)+\varphi(y) \leq b$ implies

$$
\begin{aligned}
& \psi(d(T x, T y))+\varphi(T x)+\varphi(T y) \\
& \quad \leq \gamma(a, b)[\psi(d(x, y))+\varphi(x)+\varphi(y)]
\end{aligned}
$$

for each $x, y \in \overline{\mathcal{O}\left(x_{0}\right)}$ with $x \leq y$."

Then, the sequence $\left\{x_{n}\right\}$ defined by $x_{n+1}=T x_{n}, n=$ $0,1,2, \ldots$, is Cauchy in $X$. Let $\lim _{n \rightarrow \infty} x_{n}=z$. Suppose that either (a) or (b) of Theorem 11 holds, then $z$ is a fixed point of $T$ and $\varphi(z)=0$.

Proof. Since inequality (40) implies inequality (6); the conclusion of the corollary follows from Theorem 11.

Corollary 14. Let $(X, \preceq)$ be a partially ordered set. Suppose that $T: X \rightarrow X$ is a non-decreasing selfmap on $X$. Assume that there is a metric d on $X$ such that $X$ is T-orbitally complete. Assume that there exists $x_{0} \in X$ such that $x_{0} \preceq T x_{0}$. Suppose that there exist a lower semi-continuous function $\varphi: X \rightarrow$ $[0, \infty), \psi \in \Psi$ such that the following condition holds.

"For each $0 \leq a<b<\infty$, there exist $\alpha(a, b), \beta(a, b) \in$ $[0,1)$ with $\alpha(a, b)+\beta(a, b)<1$ such that $a \leq \psi(d(x, y))+$ $\varphi(x)+\varphi(y) \leq b$ implies

$$
\begin{aligned}
\psi(d & (T x, T y))+\varphi(T x)+\varphi(T y) \\
\leq & \alpha(a, b)[\psi(d(x, T x))+\varphi(x)+\varphi(T x)] \\
& +\beta(a, b)[\psi(d(y, T y))+\varphi(y)+\varphi(T y)]
\end{aligned}
$$

for each $x, y \in \overline{\mathcal{O}\left(x_{0}\right)}$ with $x \leq y$."

Then, the sequence $\left\{x_{n}\right\}$ defined by $x_{n+1}=T x_{n}, n=$ $0,1,2, \ldots$, is Cauchy in $X$. Let $\lim _{n \rightarrow \infty} x_{n}=z$. Suppose that either (a) or (b) of Theorem 11 holds, then $z$ is a fixed point of $T$ and $\varphi(z)=0$.

Proof. Inequality (41) implies inequality (6). Hence, the conclusion follows from Theorem 11.

As an immediate consequence, we obtain the following corollary.

Corollary 15. Let $(X, \preceq)$ be a partially ordered set. Suppose that $T: X \rightarrow X$ is a non-decreasing selfmap on $X$. Assume that there is a metric $d$ on $X$ such that $X$ is T-orbitally complete. Assume that there exists $x_{0} \in X$ such that $x_{0} \leq T x_{0}$. Suppose that there exist a lower semi-continuous function $\varphi: X \rightarrow$ $[0, \infty), \psi \in \Psi$, and $\alpha, \beta \in[0,1)$ with $\alpha+\beta<1$ such that

$$
\begin{aligned}
\psi(d & (T x, T y))+\varphi(T x)+\varphi(T y) \\
\leq & \alpha[\psi(d(x, T x))+\varphi(x)+\varphi(T x)] \\
& +\beta[\psi(d(y, T y))+\varphi(y)+\varphi(T y)]
\end{aligned}
$$

for each $x, y \in \overline{\mathcal{O}\left(x_{0}\right)}$ with $x \leq y$.

Then, the sequence $\left\{x_{n}\right\}$ defined by $x_{n+1}=T x_{n}, n=$ $0,1,2, \ldots$, is Cauchy in $X$. Let $\lim _{n \rightarrow \infty} x_{n}=z$. Suppose that either (a) or (b) of Theorem 11 holds, then $z$ is a fixed point of $T$ and $\varphi(z)=0$.

Corollary 16. Let $(X, \preceq)$ be a partially ordered set. Suppose that $T: X \rightarrow X$ is a non-decreasing selfmap on $X$. Assume that there is a metric $d$ on $X$ such that $X$ is T-orbitally complete. Assume that there exists $x_{0} \in X$ such that $x_{0} \leq T x_{0}$. Suppose that there exist a lower semi-continuous function $\varphi: X \rightarrow$ $[0, \infty), \psi \in \Psi$ such that the following condition holds.

"For each $0 \leq a<b<\infty$ there exist $\alpha(a, b), \beta(a, b)$, and $\gamma(a, b) \in[0,1)$ with $\alpha(a, b)+\beta(a, b)+\gamma(a, b)<1$ such that $a \leq \psi(d(x, y))+\varphi(x)+\varphi(y) \leq b$ implies

$$
\begin{aligned}
\psi(d & (T x, T y))+\varphi(T x)+\varphi(T y) \\
\leq & \alpha(a, b)[\psi(d(x, y))+\varphi(x)+\varphi(y)] \\
& +\beta(a, b)[\psi(d(x, T x))+\varphi(x)+\varphi(T x)] \\
& +\gamma(a, b)[\psi(d(y, T y))+\varphi(y)+\varphi(T y)]
\end{aligned}
$$

for each $x, y \in \overline{\mathcal{O}\left(x_{0}\right)}$ with $x \preceq y$."

Then, the sequence $\left\{x_{n}\right\}$ defined by $x_{n+1}=T x_{n}, n=$ $0,1,2, \ldots$, is Cauchy in X. Let $\lim _{n \rightarrow \infty} x_{n}=z$. Suppose that either (a) or (b) of Theorem 11 holds, then $z$ is a fixed point of $T$ and $\varphi(z)=0$.

Proof. The conclusion follows from Theorem 11, since inequality (43) implies inequality (6).

We now obtain the following corollary from Corollary 16.

Corollary 17. Let $(X, \preceq)$ be a partially ordered set and $d$ a metric on $X$. Suppose that $T: X \rightarrow X$ is a non-decreasing selfmap on $X$. Assume that there is a metric $d$ on $X$ such that $X$ is T-orbitally complete. Assume that there exists $x_{0} \in X$ such that $x_{0} \preceq T x_{0}$. Suppose that there exist a lower semicontinuous function $\varphi: X \rightarrow[0, \infty), \psi \in \Psi$ and there exist $\alpha, \beta$, and $\gamma \in[0,1)$ with $\alpha+\beta+\gamma<1$ such that

$$
\begin{aligned}
\psi(d & (T x, T y))+\varphi(T x)+\varphi(T y) \\
\leq & \alpha[\psi(d(x, y))+\varphi(x)+\varphi(y)] \\
& +\beta[\psi(d(x, T x))+\varphi(x)+\varphi(T x)] \\
& +\gamma[\psi(d(y, T y))+\varphi(y)+\varphi(T y)]
\end{aligned}
$$

for each $x, y \in \overline{\mathcal{O}\left(x_{0}\right)}$ with $x \preceq y$. 
Then, the sequence $\left\{x_{n}\right\}$ defined by $x_{n+1}=T x_{n}, n=0,1$, $2, \ldots$, is Cauchy in X. Let $\lim _{n \rightarrow \infty} x_{n}=z$. Suppose that either (a) or (b) of Theorem 11 holds, then $z$ is a fixed point of $T$ and $\varphi(z)=0$.

Remark 18. By choosing $\psi(t)=t$ in Corollaries 13,15 , and 17 , we obtain partially ordered versions of Theorems 1, 2, and 3, respectively.

Remark 19. By choosing $\varphi \equiv 0$ and $\psi(t)=t, t \geq$ 0 , in Corollary 12, we obtain Theorem 5 as a corollary to Corollary 12, which in turn Theorem 5 follows as a corollary to Theorem 11.

Theorem 20. Let $(X, \preceq)$ be a partially ordered set. Suppose that $T_{1}: X \rightarrow X$ is a non-decreasing selfmap on $X$. Assume that there is a metric $d$ on $X$ such that $X$ is $T_{1}$-orbitally complete. Assume that there exists $x_{0} \in X$ such that $x_{0} \preceq T_{1} x_{0}$. Let $\left\{T_{i} / T_{i}: X \rightarrow X, i=1,2,3, \ldots\right\}$ be a sequence of maps and suppose that there exist a lower semi-continuous function $\varphi: X \rightarrow[0, \infty), \psi \in \Psi$, and $k \in[0,1)$ such that

$$
\psi\left(d\left(T_{1} x, T_{j} y\right)\right)+\varphi\left(T_{1} x\right)+\varphi\left(T_{j} y\right) \leq k M(x, y),
$$

where

$$
\begin{aligned}
& M(x, y) \\
& =\max \{\psi(d(x, y))+\varphi(x)+\varphi(y), \\
& \psi\left(d\left(x, T_{1} x\right)\right)+\varphi(x)+\varphi\left(T_{1} x\right), \\
& \left.\psi\left(d\left(y, T_{j} y\right)\right)+\varphi(y)+\varphi\left(T_{j} y\right)\right\}
\end{aligned}
$$

for each $x, y \in \overline{\mathcal{O}\left(x_{0}\right)}$ with $x \preceq y$ and $j=1,2,3, \ldots$.

If either $(a)$ or $(b)$ of Theorem 11 holds, then the family of maps $\left\{T_{j} / j=1,2,3, \ldots\right\}$ has a common fixed point.

Proof. If $j=1$, then $T_{1}$ has a fixed point by Corollary 12. Let that fixed point be $u$ (say). Then, $\varphi(u)=0$. We show that $T_{j} u=u$ for each $j=2,3,4, \ldots$.. Let $j>1$ be fixed such that $T_{j} u \neq u$.

Now, using (45), we get

$$
\psi\left(d\left(T_{1} u, T_{j} u\right)\right)+\varphi\left(T_{1} u\right)+\varphi\left(T_{j} u\right) \leq k M(u, u),
$$

where

$$
\begin{aligned}
& M(u, u) \\
& =\max \{\psi(d(u, u))+\varphi(u)+\varphi(u), \\
& \psi\left(d\left(u, T_{1} u\right)\right)+\varphi(u)+\varphi\left(T_{1} u\right), \\
& \left.\psi\left(d\left(u, T_{j} u\right)\right)+\varphi(u)+\varphi\left(T_{j} u\right)\right\} \\
& =\psi\left(d\left(u, T_{j} u\right)\right)+\varphi\left(T_{j} u\right) .
\end{aligned}
$$

Then, from (47), we obtain

$$
\begin{aligned}
& \psi\left(d\left(T_{1} u, T_{j} u\right)\right)+\varphi\left(T_{j} u\right) \\
& \quad \leq k\left[\psi\left(d\left(T_{1} u, T_{j} u\right)\right)+\varphi\left(T_{j} u\right)\right],
\end{aligned}
$$

Thus, the conclusion of the theorem follows.

The following is an example in support of Theorem 11.

Example 21. Let $X=[0,2)$ with the usual metric. We define a partial order $\preceq$ on $X$ by

$$
\begin{gathered}
\preceq:=\{(x, y): x, y \in[0,1), x \geq y\} \\
\cup\{(x, y): x, y \in[1,2), x=y\} .
\end{gathered}
$$

We define $T: X \rightarrow X, \psi:[0, \infty) \rightarrow[0, \infty)$, and $\varphi: X \rightarrow$ $[0, \infty)$ by

$$
\begin{gathered}
T(x)= \begin{cases}\frac{x}{2}, & \text { if } x \in[0,1), \\
\frac{1+x}{2}, & \text { if } x \in[1,2),\end{cases} \\
\psi(t)=\frac{t}{2}, \quad t \geq 0, \\
\varphi(x)= \begin{cases}\frac{x}{2}, & \text { if } 0 \leq x \leq 1, \\
\frac{3}{4} x, & \text { if } 1<x<2 .\end{cases}
\end{gathered}
$$

Let $x_{0}=1 / 4$; then, $\mathcal{O}\left(x_{0}\right)=\left\{\left(1 / 2^{n}\right) / n \geq 2, n \in Z^{+}\right\}$and $\overline{\mathcal{O}\left(x_{0}\right)}=\mathscr{O}\left(x_{0}\right) \cup\{0\}$.

And $T x_{0}=1 / 8$ so that $x_{0} \preceq T x_{0}$.

We now verify inequality (6).

In this example, we choose $\gamma(a, b)=3 / 4$ for each $a, b \in$ $[0, \infty), 0 \leq a<b$ satisfying $a \leq \psi(d(x, y))+\varphi(x)+\varphi(y) \leq b$ for all $x, y \in \overline{\mathcal{O}\left(x_{0}\right)}$.

Let $x, y \in \overline{\mathcal{O}\left(x_{0}\right)}$.

Case (i) $\left(x=1 / 2^{n}, y=1 / 2^{m}\right.$, and $\left.n \geq m \geq 2\right)$. Suppose that $a \leq \psi(d(x, y))+\varphi(x)+\varphi(y) \leq b$. That is, $a \leq(1 / 2)\left[\left(1 / 2^{m}\right)-\right.$ $\left.\left(1 / 2^{n}\right)\right]+\left(1 / 2^{n+1}\right)+\left(1 / 2^{m+1}\right) \leq b$. That is, $a \leq 1 / 2^{m} \leq b$. Now,

$$
\begin{aligned}
& \psi(d(T x, T y))+\varphi(T x)+\varphi(T y) \\
& \quad=\frac{1}{2^{m+1}} \\
& \quad \leq \frac{3}{4} \frac{1}{2^{m}}=\gamma(a, b)[\psi(d(x, y))+\varphi(x)+\varphi(y)] \\
& \quad \leq \gamma(a, b) M(x, y) .
\end{aligned}
$$

Case (ii) ( $x=1 / 2^{n}, n \geq 2$, and $\left.y=0\right)$. Suppose that $a \leq$ $\psi(d(x, y))+\varphi(x)+\varphi(y) \leq b$; that is, $a \leq 1 / 2^{n} \leq b$. Now,

$$
\begin{aligned}
& \psi(d(T x, T y))+\varphi(T x)+\varphi(T y) \\
& \quad=\frac{1}{2^{n+1}} \\
& \quad \leq \frac{3}{4} \frac{1}{2^{n}}=\gamma(a, b)[\psi(d(x, y))+\varphi(x)+\varphi(y)] \\
& \quad \leq \gamma(a, b) M(x, y) .
\end{aligned}
$$


Case (iii) $\left(x=y=1 / 2^{n}, n \geq 2\right)$. Suppose that $a \leq \psi(d(x, y))+$ $\varphi(x)+\varphi(y) \leq b$; that is, $a \leq 1 / 2^{n} \leq b$. Now,

$$
\psi(d(T x, T y))+\varphi(T x)+\varphi(T y)=\frac{1}{2^{n+1}} .
$$

Hence the verification of inequality (6) is the same as in Case (ii).

Case (iv) $(x=y=0)$. In this case, $\psi(d(T x, T y))+\varphi(T x)+$ $\varphi(T y)=0$ so that inequality (6) holds trivially.

From all the above cases, it is clear that inequality (6) holds for all $x, y \in \overline{\mathcal{O}\left(x_{0}\right)}$. Hence, all the hypotheses of Theorem 11 hold and 0 is the fixed point of $T$ in $\overline{\mathcal{O}\left(x_{0}\right)}$. Also, $\varphi(0)=0$.

Now, for $x=0$ and $y=1$, suppose that there exists $0 \leq$ $a<b<\infty$ such that $a \leq \psi(d(x, y))+\varphi(x)+\varphi(y)=\psi(1)+$ $\varphi(0)+\varphi(1)<b$. Then, we have

$$
\begin{aligned}
\psi( & (T(T x, T y))+\varphi(T x)+\varphi(T y) \\
& =\psi(1)+\varphi(0)+\varphi(1) \\
& \quad \neq \gamma(a, b)[\psi(d(x, y))+\varphi(x)+\varphi(y)]
\end{aligned}
$$

so that inequality (1) fails to hold for any $\varphi$ and $\gamma(a, b) \in[0,1)$. Hence Theorem 1 is not applicable.

Example 22. Let $X=[0,1)$ with the usual metric. We define a partial order $\preceq$ on $X$ by

" $x \preceq y$ if and only if $x \geq y$ for each $x, y \in X$."

We define $T: X \rightarrow X, \psi:[0, \infty) \rightarrow[0, \infty)$, and $\varphi:$ $X \rightarrow[0, \infty)$ by

$$
\begin{gathered}
T(x)= \begin{cases}0, & \text { if } x=0, \\
\frac{1}{4}, & \text { if } \frac{1}{2} \leq x \leq 1, \\
\frac{3}{16}, & \text { if } \frac{1}{4} \leq x<\frac{1}{2}, \\
\frac{3}{2} \frac{1}{2^{n+1}}, & \text { if } \frac{1}{2^{n+1}} \leq x<\frac{1}{2^{n}}, \quad n \geq 2,\end{cases} \\
\varphi(t)=2 t, \quad t \geq 0, \\
\varphi(x)= \begin{cases}\frac{x}{6}, & \text { if } 0 \leq x<\frac{3}{16}, \\
x-\frac{3}{16}, & \text { if } \frac{3}{16} \leq x<1 .\end{cases}
\end{gathered}
$$

Let $x_{0}=2 / 3$. Then, $T x_{0}=1 / 4$ so that $x_{0} \leq T x_{0}$. Consider

$$
O\left(x_{0}\right)=\left\{\frac{2}{3}, \frac{1}{4}, \frac{3}{16}, \frac{3}{16}, \frac{3}{16}, \ldots\right\}, \quad \overline{O\left(x_{0}\right)}=O\left(x_{0}\right) .
$$

We now verify inequality (6).

We choose $\gamma(a, b)=12 / 69$ for each $0 \leq a<b<\infty$ satisfying $a \leq \psi(d(x, y))+\varphi(x)+\varphi(y) \leq b$ for all $x, y \in \overline{\mathcal{O}\left(x_{0}\right)}$.
Let $x, y \in \overline{\mathcal{O}\left(x_{0}\right)}$.

Case (i) $(x=2 / 3, y=1 / 4)$. Suppose that $a \leq \psi(d(x, y))+$ $\varphi(x)+\varphi(y) \leq b$; that is, $a \leq 11 / 8 \leq b$. Now,

$$
\begin{gathered}
M(x, y) \\
=\max \{\psi(d(x, y))+\varphi(x)+\varphi(y), \\
\psi(d(x, T x))+\varphi(x)+\varphi(T x), \\
\psi(d(y, T y))+\varphi(y)+\varphi(T y)\} \\
=\max \left\{\frac{11}{8}, \frac{11}{8}, \frac{3}{16}\right\}=\frac{11}{8}, \\
\psi(d(T x, T y))+\varphi(T x)+\varphi(T y) \\
=\frac{3}{16}<\frac{12}{69} \frac{11}{8}=\gamma(a, b) M(x, y) .
\end{gathered}
$$

Case (ii) $(x=2 / 3, y=3 / 16)$. Suppose that $a \leq \psi(d(x, y))+$ $\varphi(x)+\varphi(y) \leq b$; that is, $a \leq 69 / 48 \leq b$. Now,

$$
\begin{gathered}
\psi(d(T x, T y))+\varphi(T x)+\varphi(T y) \\
=\psi\left(\frac{1}{16}\right)+\varphi\left(\frac{1}{4}\right)+\varphi\left(\frac{3}{16}\right)=\frac{3}{16}, \\
M(x, y) \\
=\max \{\psi(d(x, y))+\varphi(x)+\varphi(y), \\
\psi(d(x, T x))+\varphi(x)+\varphi(T x), \\
\psi(d(y, T y))+\varphi(y)+\varphi(T y)\} \\
=\max \left\{\frac{69}{48}, \frac{11}{8}, 0\right\}=\frac{69}{48}
\end{gathered}
$$

so that

$$
\psi(d(T x, T y))+\varphi(T x)+\varphi(T y) \leq \gamma(a, b) M(x, y) .
$$

Case (iii) $(x=1 / 4, y=3 / 16)$. Suppose that $a \leq \psi(d(x, y))+$ $\varphi(x)+\varphi(y) \leq b$; that is, $a \leq 3 / 16 \leq b$. Now,

$$
\begin{aligned}
& \psi(d(T x, T y))+\varphi(T x)+\varphi(T y) \\
& \quad=\psi(0)+\varphi\left(\frac{3}{16}\right)+\varphi\left(\frac{3}{16}\right)=0 .
\end{aligned}
$$

Hence, (6) holds trivially. 
Case (iv) $(x=y=2 / 3)$. Suppose that $a \leq \psi(d(x, y))+\varphi(x)+$ $\varphi(y) \leq b$; that is, $a \leq 23 / 24 \leq b$. Now,

$$
\begin{gathered}
\psi(d(T x, T y))+\varphi(T x)+\varphi(T y) \\
=\psi(0)+\varphi\left(\frac{1}{4}\right)+\varphi\left(\frac{1}{4}\right)=\frac{1}{8}, \\
M(x, y) \\
=\max \{\psi(d(x, y))+\varphi(x)+\varphi(y), \\
\psi \psi(d(x, T x))+\varphi(x)+\varphi(T x), \\
=\max \left\{\frac{23}{24}, \frac{11}{8}, \frac{11}{8}\right\}=\frac{11}{8} .
\end{gathered}
$$

Then,

$$
\begin{gathered}
\psi(d(T x, T y))+\varphi(T x)+\varphi(T y) \\
=\frac{1}{8}<\frac{12}{69} \frac{11}{8}=\gamma(a, b) M(x, y) .
\end{gathered}
$$

Case (v). (let $x=y=1 / 4)$. Suppose that $a \leq \psi(d(x, y))+$ $\varphi(x)+\varphi(y) \leq b$; that is, $a \leq 1 / 8 \leq b$. Now,

$$
\begin{aligned}
& \psi(d(T x, T y))+\varphi(T x)+\varphi(T y) \\
& \quad=\psi(0)+\varphi\left(\frac{3}{16}\right)+\varphi\left(\frac{3}{16}\right)=0 .
\end{aligned}
$$

Hence, (6) holds trivially.

Hence, all the hypotheses of Theorem 11 hold and 3/16 is the fixed point of $T$ in $\overline{\mathcal{O}\left(x_{0}\right)}$. Also, $\varphi(3 / 16)=0$.

We observe that at $x=0$ and $y=3 / 16$,

$$
\begin{gathered}
\psi(d(T x, T y))+\varphi(T x)+\varphi(T y) \\
=\psi\left(\frac{3}{16}\right)+\varphi(0)+\varphi\left(\frac{3}{16}\right), \\
\psi(d(x, y))+\varphi(x)+\varphi(y) \\
=\psi\left(\frac{3}{16}\right)+\varphi(0)+\varphi\left(\frac{3}{16}\right), \\
\psi(d(x, T x))+\varphi(x)+\varphi(T x) \\
\quad+\psi(d(y, T y))+\varphi(y)+\varphi(T y) \\
=2\left[\varphi(0)+\varphi\left(\frac{3}{16}\right)\right] .
\end{gathered}
$$

Hence, inequality (2) does not hold for any $\varphi, \psi$, and $\gamma \in$ $[0,1 / 2)$. Therefore, Theorem 2 is not applicable.

Also, $\psi(d(T x, T y))=\psi(3 / 16)$ and $\psi(d(x, y))=3 / 16$. Hence, $\psi(d(T x, T y)) \leq k \psi(d(x, y))$ does not hold for any $\psi \in$ $\Psi$ and $k \in[0,1)$. Thus, inequality (4) fails to hold, and hence Theorem 5 is not applicable.

Thus, by Remark 19, we conclude that Theorem 11 is a generalization of Theorem 5 .

\section{Acknowledgment}

The authors thank the referee for his/her valuable suggestions, which improved the presentation of the paper.

\section{References}

[1] S. Banach, "Sur les operations dans les ensembles abstraits at leur application aux equaltions integrales," Fundamenta Mathematicae, vol. 3, pp. 173-181, 1922.

[2] V. Berinde and F. Vetro, "Common fixed points of mappings satisfying implicit contractive conditions," Fixed Point Theory and Applications, vol. 2012, article 105, 2012.

[3] S. K. Chatterjea, "Fixed-point theorems," Comptes Rendus de l'Académie Bulgare des Sciences, vol. 25, pp. 727-730, 1972.

[4] L. B. Ćirić, "A generalization of Banach's contraction principle," Proceedings of the American Mathematical Society, vol. 45, pp. 267-273, 1974.

[5] R. Kannan, "Some results on fixed points," Bulletin of the Calcutta Mathematical Society, vol. 60, pp. 71-76, 1960.

[6] R. Kannan, "Some results on fixed points-II," The American Mathematical Monthly, vol. 76, pp. 405-408, 1969.

[7] S. Reich, "Kannan's fixed point theorem," Bollettino della Unione Matematica Italiana, vol. 4, no. 4, pp. 1-11, 1971.

[8] B. Samet, C. Vetro, and F. Vetro, "From metric spaces to partial metric spaces," Fixed Point Theory and Applications, vol. 2013, article 5, 2013.

[9] A. C. M. Ran and M. C. B. Reurings, "A fixed point theorem in partially ordered sets and some applications to matrix equations," Proceedings of the American Mathematical Society, vol. 132, no. 5, pp. 1435-1443, 2004.

[10] D. Türkoğlu, O. Özer, and B. Fisher, "Fixed point theorems for T-orbitally complete spaces," Mathematica, no. 9, pp. 211-218, 1999.

[11] H.-S. Ding, Z. Kadelburg, and H. K. Nashine, "Common fixed point theorems for weakly increasing mappings on ordered orbitally complete metric spaces," Fixed Point Theory and Applications, vol. 2012, article 85, 2012.

[12] H. K. Nashine, Z. Kadelburg, and Z. Golubović, "Common fixed point results using generalized altering distances on orbitally complete ordered metric spaces," Journal of Applied Mathematics, vol. 2012, Article ID 382094, 12 pages, 2012.

[13] G. V. R. Babu and P. D. Sailaja, "A fixed point theorem of generalized weakly contractive maps in orbitally complete metric spaces," Thai Journal of Mathematics, vol. 9, no. 1, pp. 1$10,2011$. 


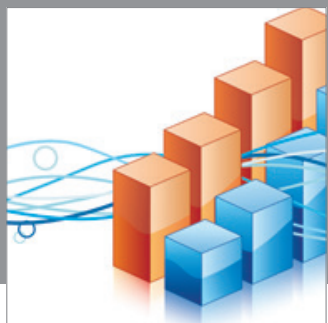

Advances in

Operations Research

mansans

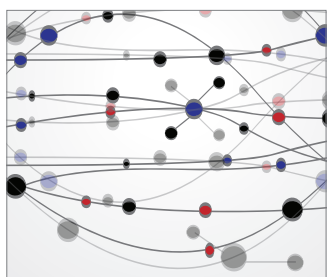

The Scientific World Journal
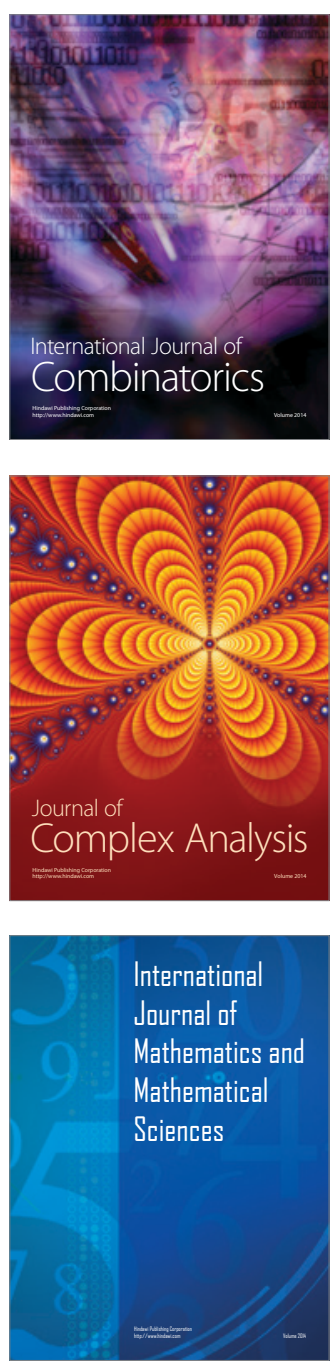
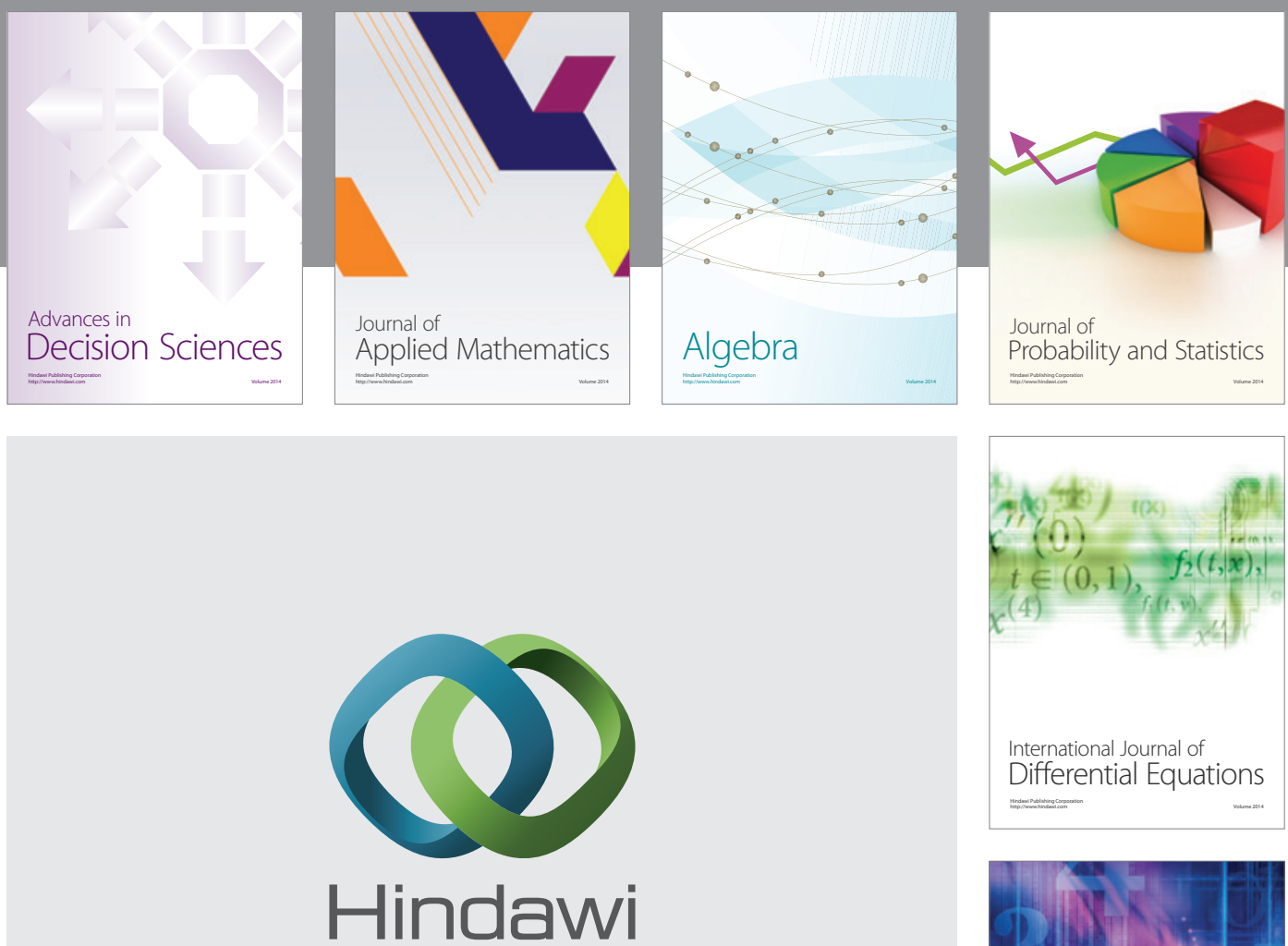

Submit your manuscripts at http://www.hindawi.com
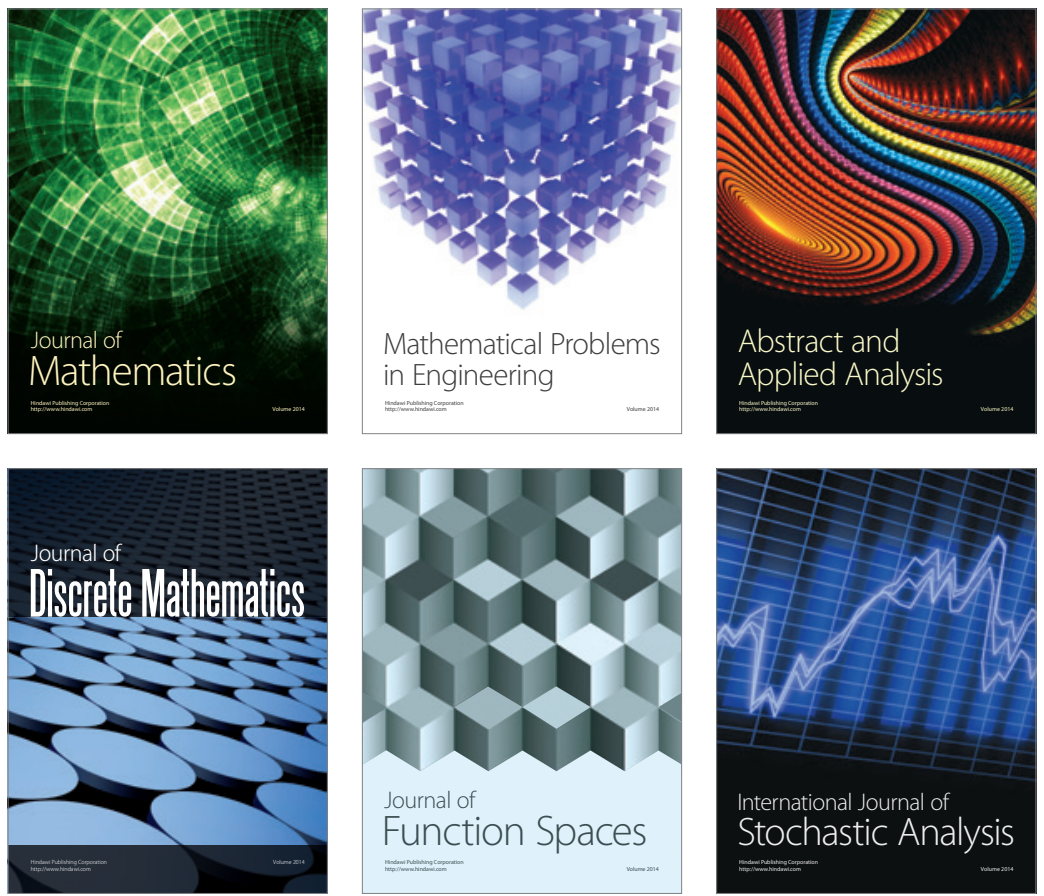

Journal of

Function Spaces

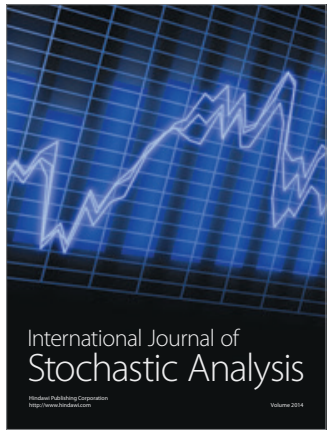

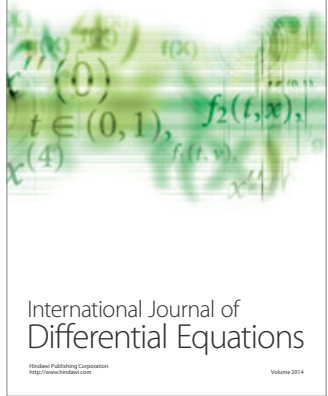
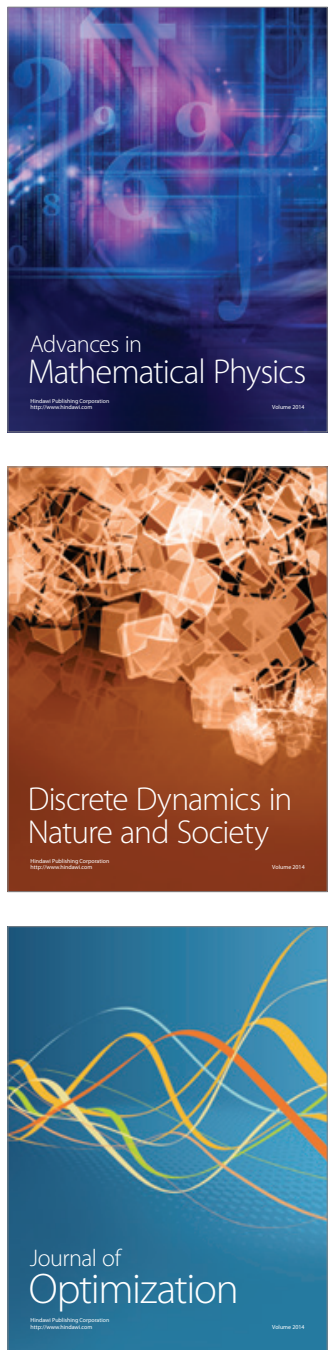